TRANSACTIONS OF THE

AMERICAN MATHEMATICAL SOCIETY

Volume 355, Number 1, Pages 155-167

S 0002-9947(02)03115-X

Article electronically published on September 6, 2002

\title{
ON HYPERSPHERICITY OF MANIFOLDS WITH FINITE ASYMPTOTIC DIMENSION
}

\author{
A. N. DRANISHNIKOV
}

\begin{abstract}
We prove the following embedding theorems in the coarse geometry:

Theorem A. Every metric space $X$ with bounded geometry whose asymptotic dimension does not exceed $n$ admits a large scale uniform embedding into the product of $n+1$ locally finite trees.

Corollary. Every metric space $X$ with bounded geometry whose asymptotic dimension does not exceed $n$ admits a large scale uniform embedding into a non-positively curved manifold of dimension $2 n+2$.
\end{abstract}

The Corollary is used in the proof of the following.

Theorem B. For every uniformly contractible manifold $X$ whose asymptotic dimension is finite, the product $X \times \mathbf{R}^{n}$ is integrally hyperspherical for some $n$.

Theorem B together with a theorem of Gromov-Lawson implies the result, previously proven by G. Yu (1998), which states that an aspherical manifold whose fundamental group has a finite asymptotic dimension cannot carry a metric of positive scalar curvature.

We also prove that if a uniformly contractible manifold $X$ of bounded geometry is large scale uniformly embeddable into a Hilbert space, then $X$ is stably integrally hyperspherical.

\section{INTRODUCTION}

Gromov introduced several notions of largeness of Riemannian manifolds. For example, a manifold $X$ that is a universal cover of a closed aspherical manifold $M^{n}$ with the fundamental group $\Gamma=\pi_{1}\left(M^{n}\right)$, supplied with a $\Gamma$-invariant metric is large in the sense that it is uniformly contractible. We recall that $X$ is uniformly contractible if there is a function $S(r)$ such that for any $x \in X$, every ball $B_{r}(x)$ of radius $r$ centered at $x$ is contractible to a point in the ball $B_{S(r)}(x)$. For the purpose of the Novikov conjecture and other related conjectures, it is important to show that universal covers of aspherical manifolds are large in some cohomological sense. Perhaps the weakest property of this type is hypersphericity.

Received by the editors January 23, 2001 and, in revised form, May 20, 2002.

2000 Mathematics Subject Classification. Primary 53C23.

Key words and phrases. Hyperspherical manifold, uniform embedding, asymptotic dimension, scalar curvature, Gromov-Lawson conjecture.

The author was partially supported by NSF grant DMS-9971709. 
Definition $([\mathrm{G}-\mathrm{L}])$. An $n$-dimensional manifold $X$ is called hyperspherical if for every $\epsilon>0$, there is a $\epsilon$-contracting proper map $f_{\epsilon}: X \rightarrow S^{n}$ of nonzero degree onto the standard unit $n$-sphere.

Here a continuous map $f: X \rightarrow S^{n}$ is called proper if it has only one unbounded preimage. Gromov and Lawson proved the following G-L].

Gromov-Lawson's Theorem. An aspherical manifold with a hyperspherical universal cover cannot carry a metric of positive scalar curvature.

Then the natural question appeared G2]:

Problem 1. Is every uniformly contractible manifold hyperspherical?

This problem is still open and even new examples of Gromov [G4] leave a possibility for a positive answer. We have defined here the notion of rational hypersphericity. One can define integral hypersphericity by taking into account only maps of degree one. A further generalization consists of replacing the $n$-sphere by $\mathbf{R}^{n}$. It leads to the notion of hypereuclidean manifolds G2. In the integral case, these two properties seem to be equivalent. An example of a uniformly contractible manifold that is not integrally hypereuclidean was constructed in [D-F-W]. We note that this example is rationally hypereuclidean.

The following definition is due to Gromov [G1]:

Definition. A map $f: X \rightarrow Y$ between metric spaces is called a large scale uniform embedding if there are two functions $\rho_{1}, \rho_{2}:[0, \infty) \rightarrow[0, \infty)$ tending to infinity such that

$$
\rho_{1}\left(d_{X}\left(x, x^{\prime}\right)\right) \leq d_{Y}\left(f(x), f\left(x^{\prime}\right)\right) \leq \rho_{2}\left(d_{X}\left(x, x^{\prime}\right)\right) \text { for all } x, x^{\prime} \in X .
$$

It defines an embedding in the coarse category introduced by Roe [Ro1].

In Section 2 of this paper we prove the following.

Theorem 1. Suppose that a uniformly contractible manifold $X$ admits a large scale uniform embedding into $\mathbf{R}^{n}$ with $n>2 \operatorname{dim} X$. Then $X \times \mathbf{R}^{n}$ is integrally hyperspherical.

Theorem 1 can be generalized:

Theorem 1'. Suppose that a uniformly contractible manifold $X$ admits a large scale uniform embedding into a simply connected non-positively curved n-dimensional manifold with $n>2 \operatorname{dim} X$. Then $X \times \mathbf{R}^{n}$ is integrally hyperspherical.

Corollary. Let $M$ be a closed aspherical manifold and assume that the fundamental group $\Gamma=\pi_{1}(M)$ as a metric space with the word metric admits a large scale uniform embedding into a simply connected non-positively curved n-dimensional manifold. Then $M$ cannot carry a metric with positive scalar curvature.

Some results of this type were predicted by Gromov (see his remark (b), page 183 of [G3]).

In this paper we prove an embedding theorem (Theorem 4) in the coarse category, which enables us to prove the following result (Theorem 5).

For every uniformly contractible manifold $X$ with $\operatorname{asdim} X \leq k$, the space $X \times \mathbf{R}^{n}$ is integrally hyperspherical for $n=2 k+3$. 
Corollary. Let $M$ be a closed aspherical manifold and assume that the fundamental group $\Gamma=\pi_{1}(M)$ as a metric space with the word metric has finite asymptotic dimension asdim $\Gamma<\infty$. Then $M$ cannot carry a metric with a positive scalar curvature.

This Corollary also follows from the theorems of Yu [Yu1, [Yu2].

The First Theorem of Yu. If a finitely presented group $\Gamma$ has a finite asymptotic dimension, in particular, if it is large scale uniformly embeddable in $\mathbf{R}^{n}$, then the coarse Baum-Connes conjecture holds for $\Gamma$.

The Second Theorem of Yu. If a finitely presented group $\Gamma$ can be large scale uniformly embedded into a Hilbert space, then the coarse Baum-Connes conjecture holds for $\Gamma$.

It is known that The Second Theorem of Yu implies The First [H-R2]. We refer for the formulation of the coarse Baum-Connes conjecture to Ro1, Ro2. Here we note that it has been disproved recently by Higson and others by means of expanders which were brought to this area by Gromov [G4].

One can say that all open Riemanian manifolds that obey the coarse BaumConnes conjecture are large in some refined sense. This largeness is related to the hypersphericity and the hypereuclideaness though it is different. The connection between them is based of the fact [Ro1] that integrally (rationally) hypereuclidean manifolds satisfy the injectivity part of the (rational) coarse Baum-Connes conjecture. We note that the injectivity part of the coarse Baum-Connes conjecture is not disproved by the recent examples, and note that the injectivity is sufficient for proving of the Gromov-Lawson conjecture about nonexistence of a positive scalar curvature metric on a closed aspherical Reimannian manifold.

Both of Yu's theorems hold for all proper metric spaces with bounded geometry. We recall that a metric space $X$ is said to have bounded geometry if for every $\epsilon>0$ and every $r>0$, there is a $c$ such that the $\epsilon$-capacity of every $r$-ball $B_{r}(x)$ does not exceed $c$. The latter means that a ball $B_{r}(x)$ contains no more than $c \epsilon$-disjoint points.

Definition. An $n$-dimensional manifold $X$ is called stably (integrally) hyperspherical if for any $\epsilon>0$, there is an $m$ such that $X \times \mathbf{R}^{m}$ admits an $\epsilon$-contracting proper map of nonzero degree (of degree one) onto the unit $(n+m)$-sphere $S^{n+m}$.

We prove the following.

Theorem 2. Suppose that $X$ is a uniformly contractible manifold with bounded geometry and assume that $X$ admits a large scale uniform embedding into a Hilbert space. Then $X$ is stably integrally hyperspherical.

It is unclear whether stable hypersphericity implies the Gromov-Lawson conjecture. A positive answer to the following problem would give a simple argument for the implication: Stable hypersphericity $\Rightarrow$ Gromov-Lawson.

Problem 2. Does there exist a positive constant $c>0$ such that the $K$-area of the unit $2 n$-sphere is greater than $c$ for all $n$ ?

We recall the definition of $K$-area from [G3]. For an even-dimensional Riemannian manifold $M$, the $K$-area is

$$
K \text {-area }(M)=\left(\inf _{X}\{\|R(X)\|\}\right)^{-1},
$$


where the infimum is taken over all Hermitian vector bundles $V$ on $M$ endowed with Hermitian connections and with some of the Chern numbers nonzero, and $R(V)$ is the curvature of $V$. Since, by the definition, $R(V)$ is a section of the bundle $\operatorname{Hom}\left(V,\left(\bigwedge^{2} \tau_{\mathbf{C}}^{*}\right) \otimes V\right)$, where $\tau_{\mathbf{C}}^{*}$ is the complexification of the cotangent bundle of $M$, the curvature $R(V)$ can be viewed as a 2-form on $M$ taking values in the space of endomorphisms $\operatorname{Hom}(V, V)$, equipped with the norm:

$$
\|A\|=\sup _{\|x\|=1}\|A x\|_{V}, \quad A: V \rightarrow V .
$$

Finally, $\|R(V)\|=\sup \{\|R(V)(a \wedge b)\|\}$, where the supremum is taken over all orthonormal bivectors $a \wedge b$ in the tangent bundle of $M$. The definition of $K$-area can be extended to open manifolds by taking bundles $V$ trivialized at infinity. To take care of odd-dimensional manifolds, Gromov defines the stabilized $K$-area of a manifold $M$ as

$$
K-\operatorname{area}_{\text {st }}(M)=\sup _{m}\left\{K-\operatorname{area}\left(M \times \mathbf{R}^{m}\right)\right\} .
$$

The main fact about the $K$-area is the following inequality [G3]:

$$
a\left(K-\operatorname{area}_{\mathrm{st}}(X)\right) \leq c_{n}
$$

for spin $n$-dimensional manifolds with the scalar curvature $\operatorname{Sc}(X) \geq a$, where $c_{n}$ depends only on $n$.

Assume that Problem 2 has an affirmative answer. Let $N$ be a closed aspherical $n$-dimensional Riemannian manifold with the stably hyperspherical universal cover $X$ and with $\operatorname{Sc}(N) \geq a$. We shall show that $a \leq 0$. Assume the contrary: $a>0$. By the definition of stable hypersphericity, there is a number $m$ and an $\epsilon$-contracting map $f: X \times \mathbf{R}^{m} \rightarrow S^{n+m}$ of nonzero degree for $\epsilon<\sqrt{\frac{c a}{c_{n}}}$. By one of the main properties of $K$-area, there is the inequality [G3]:

$$
K \text { - area }\left(X \times \mathbf{R}^{m}\right) \geq \frac{1}{\epsilon^{2}} K \text { - area }\left(S^{n+m}\right)>\frac{c_{n}}{c a} K \text {-area }\left(S^{n+m}\right) \geq \frac{c_{n}}{a} .
$$

On the other hand, since $\operatorname{Sc}(X) \geq a$, we have a contradiction:

$$
c_{n} \geq a\left(K \text { - } \text { area }_{\text {st }}(X) \geq a\left(K \text {-area }\left(X \times \mathbf{R}^{m}\right)\right)>c_{n} .\right.
$$

The other way to derive the Gromov-Lawson conjecture from the stable hypersphericity would be by extending the notion of $K$-area over loop spaces and showing that the $K$-area of $\Omega^{\infty} \Sigma^{\infty} S^{n}$ is greater than zero.

One of the objectives of this paper was to give elementary proofs of the counterparts of Yu's theorems that one obtains by replacing the conclusion "the coarse Baum-Connes conjecture holds for $\Gamma$ " by "the universal cover of a $K(\Gamma, 1)$ manifold is hyperspherical". Theorems 1 and 2 of the present paper can be thought of as counterparts of the First and Second Theorems of $\mathrm{Yu}$, respectively. However, while Theorem 1 leads to the same range of geometric corollaries as Yu's theorems, to obtain these corollaries from Theorem 2, one needs a positive answer to Problem 2.

\section{Proofs of Theorems 1 And 2}

Since every contractible $k$-manifold $X$ is homeomorphic to $\mathbf{R}^{k+1}$ after crossing with the real line $\mathbf{R}$, without loss of generality we may assume that $X$ is homeomorphic to $\mathbf{R}^{k}$. Let $h: \mathbf{R}^{k} \rightarrow X$ be a homeomorphism. We denote by $S_{r}^{k-1}$ the standard sphere in $\mathbf{R}^{k}$ of radius $r$ with the center at 0 . Note that the family 
$h\left(S_{r}^{k-1}\right)$ tends to infinity in $X$ as $r$ approaches infinity. Let $N_{\lambda}(A)$ denote the $\lambda$ neighborhood of $A$ in an ambient space $W$. By $B_{r}^{m}$ we denote the standard $r$-ball in $\mathbf{R}^{m}$.

Lemma 1. Let $X$ be a uniformly contractible manifold with bounded geometry. Suppose that $X$ is homeomorphic to $\mathbf{R}^{k}$ and $X$ is isometrically embedded in a metric space $W$. Then for any $\lambda>0$, there is $r>0$ such that the neighborhood $N_{\lambda}\left(h\left(S_{r}^{k-1}\right)\right)$ in $W$ admits a retraction onto $h\left(S_{r}^{k-1}\right)$.

Proof. Let $S: \mathbf{R}_{+} \rightarrow \mathbf{R}_{+}$be a monotone contractibility function on $X$. Clearly, $S(t) \geq t$. Since $X$ is a space of bounded geometry, there is a uniformly bounded cover $\mathcal{U}$ on $X$ of finite multiplicity $m$ and with Lebesgue number $>4 \lambda$ (see H-R1] or $[\overline{\mathrm{Dr}})$. Let $d$ be an upper bound for the diameters of elements of the cover $\mathcal{U}$. Define $T(t)=2 S(3 t)$. Let $T^{l}$ denote the $l$ times iteration of $T$. We take $r$ such that $d\left(h(0), h\left(S_{r}^{k-1}\right)\right) \geq T^{m+k}(d)$. Let $\overline{\mathcal{U}}=\left\{U \in \mathcal{U} \mid U \cap h\left(S_{r}^{k-1}\right) \neq \emptyset\right\}$ be the restriction of $\mathcal{U}$ over $h\left(S_{r}^{k-1}\right)$. Note that $\overline{\mathcal{U}}$ covers the neighborhood $N_{\lambda}\left(h\left(S_{r}^{k-1}\right)\right)=N$. Let $\nu: N \rightarrow N(\overline{\mathcal{U}})$ be a projection to the nerve of the cover $\overline{\mathcal{U}}$. We are going to construct a map $\phi: N(\overline{\mathcal{U}}) \rightarrow X \backslash\{h(0)\}$ such that the restriction $\left.\phi \circ \nu\right|_{h\left(S_{r}^{k-1}\right)}$ is homotopic to the identity map $i d_{h\left(S_{r}^{k-1}\right)}$. Then by the Homotopy Extension Theorem, there is an extension $\beta: N \rightarrow X \backslash\{h(0)\}$ of the identity map $i d_{h\left(S_{r}^{k-1}\right)}$. Let $\gamma: \mathbf{R}^{k} \backslash\{0\} \rightarrow S_{r}^{k-1}$ be a retraction. Then we define a retraction $\alpha: N \rightarrow h\left(S_{r}^{k-1}\right)$ as $h \circ \gamma \circ h^{-1} \beta$.

We define $\phi$ on the $l$-skeleton of $N(\overline{\mathcal{U}})$ by induction on $l$ in such a way that the diameter of the image $\phi\left(\sigma^{l}\right)$ of every $l$-simplex $\sigma^{l}$ does not exceed $T^{l}(d)$. To do that, we choose points $x_{U} \in U \cap h\left(S_{r}^{k-1}\right)$ for every $U \in \overline{\mathcal{U}}$ and define $\phi\left(v_{U}\right)=x_{U}$ for every vertex $v_{U}$ in $N(\overline{\mathcal{U}})$ corresponding to an open set $U \in \overline{\mathcal{U}}$. For every edge $\left[v_{U}, v_{U^{\prime}}\right]$ we define $\phi$ on it in such a way that $\operatorname{diam} \phi\left(\left[v_{U}, v_{U^{\prime}}\right]\right) \leq \operatorname{diam}\left(B_{S\left(d\left(x_{U}, x_{U^{\prime}}\right)\right)}\left(x_{U}\right)=\right.$ $2 S\left(d\left(x_{U}, x_{U^{\prime}}\right)\right) \leq 2 S(2 d) \leq 2 S(3 d)=T(d)$. Assume that $\phi$ is defined on the $l$ skeleton, $l>1$, with the property that $\operatorname{diam} \phi(\sigma) \leq T^{l}(d)$ for all simplexes $\sigma$. Then for an arbitrary $(l+1)$-dimensional simplex $\Delta$, the image of the boundary $\phi(\partial \Delta)$ has diameter $\leq 2 T^{l}(d)$. Then we can extend $\phi$ over $\Delta$ with the diameter $\phi(\Delta) \leq 2 S\left(2 T^{l}(d)\right) \leq 2 S\left(3 T^{l}(d)\right)=T\left(T^{l}(d)\right)=T^{l+1}(d)$. A map $\phi$ constructed in this way has the property that $d(x, \phi \nu(x)) \leq d+T^{m}(d) \leq 2 T^{m}(d) \leq T^{m+1}(d)$.

Consider a small (with mesh smaller than $T^{m+1}(d)$ ) triangulation $\tau$ on $h\left(S_{r}^{k-1}\right.$ ) and the cellular complex structure on the product $h\left(S_{r}^{k-1}\right) \times I$ defined by $\tau$. Using induction one can extend the map

$$
i d_{h\left(S_{r}^{k-1}\right) \times\{0\}} \coprod \phi \nu_{h\left(S_{r}^{k-1}\right) \times\{1\}}: h\left(S_{r}^{k-1}\right) \times\{0,1\} \rightarrow X \backslash\{h(0)\}
$$

to a map $H: h\left(S_{r}^{k-1}\right) \times I \rightarrow X \backslash\{0\}$ with the diameter of the image $H(\sigma \times$ $I$ ) of every $i$-dimensional cell $\sigma \times I$ less than $T^{m+i+1}(d)$. Then $H\left(h\left(S_{r}^{k-1}\right)\right) \subset$ $N_{T^{m+k}(d)}\left(h\left(S_{r}^{k-1}\right)\right) \subset X \backslash\{h(0)\}$ by the choice of $r$. Thus, $H$ is a required homotopy.

Let $f: X \rightarrow Y$ and $g: X \rightarrow Z$ be a pair of maps. By $(f \Delta g): X \rightarrow Y \times Z$ we denote the diagonal product of $f$ and $g$, i.e., the map defined by the condition $(f \Delta g)(x)=(f(x), g(x))$. For a number $\mu$, we will denote by the same letter $\mu$ the multiplication by $\mu$ in the Euclidean space $\mathbf{R}^{n}$. Thus, $\mu: \mathbf{R}^{n} \rightarrow \mathbf{R}^{n}$ is defined by the formula $\mu(x)=\mu x$.

Lemma 2. Let $M$ be a closed smooth l-dimensional submanifold in the Euclidean space $\mathbf{R}^{n}$ with a trivial tubular $\epsilon$-neighborhood $N_{\epsilon}(M)$. Then for any number $d>\epsilon$, 
there is a number $\mu$ such that the diagonal embedding $j=\left(1_{M} \Delta \mu 1_{M}\right): M \rightarrow$ $\mathbf{R}^{n} \times \mathbf{R}^{n}$ has a regular neighborhood $N, \nu: N \rightarrow j(M)$ such that:

(1) $N$ admits a 1-Lipschitz map $h: N \rightarrow B_{d}^{2 n-l}$ that is a homeomorphism on every fiber $\nu^{-1}(y)$;

(2) $\operatorname{pr}_{1}(N) \subset N_{2 d}(M)$ where $p r_{1}: \mathbf{R}^{n} \times \mathbf{R}^{n} \rightarrow \mathbf{R}^{n}$ is the projection onto the first factor.

Proof. Let $q: N_{\epsilon}(M) \rightarrow B_{\epsilon}^{n-l}$ be a trivialization of the tubular neighborhood $N_{\epsilon}(M)$. Let $\lambda$ be its Lipschitz constant. Take $\mu=\frac{\lambda d}{\epsilon}$. We extend the embedding $j=\left(1_{M} \Delta \mu 1_{M}\right): M \rightarrow \mathbf{R}^{n} \times \mathbf{R}^{n}$ to the map $\bar{j}: \mathbf{R}^{n} \rightarrow \mathbf{R}^{n} \times \mathbf{R}^{n}$ defined as $\bar{j}(x)=(x, \mu x)$. Thus, the map $\bar{j}$ is a homothetic transformation of $\mathbf{R}^{n}$ to the space $L=\left\{(x, \mu x) \mid x \in \mathbf{R}^{n}\right\}$ with the homothety coefficient equal to $\sqrt{1+\mu^{2}}$. Therefore $j(M)$ admits a trivial tubular $\delta$-neighborhood $N_{\delta}^{\prime}$ in $L$ with $\delta=\epsilon \sqrt{1+\mu^{2}}$. We define $N$ as the product $N_{\delta}^{\prime} \times B_{d}^{n}$ isometrically realized in $L \times L^{\perp}$ where $L^{\perp}$ is the orthogonal complement of $L$ in $\mathbf{R}^{n} \times \mathbf{R}^{n}$. We consider the map $h_{1}=\left.\frac{d}{\epsilon} q \circ \bar{j}^{-1}\right|_{N_{\delta}^{\prime}}$ : $N_{\delta}^{\prime} \rightarrow B_{d}^{n-l}$. Note that $\frac{d}{\epsilon} \lambda\left(1 / \sqrt{1+\mu^{2}}\right)$ is a Lipschitz constant for $h_{1}$. Therefore $1=\frac{d}{\epsilon} \frac{\lambda}{\mu}$ is also a Lipschitz constant for $h_{1}$. Hence the map $\bar{h}=h_{1} \times i d_{B_{d}^{n}}: N \rightarrow$ $B_{d}^{n-l} \times B_{d}^{n}$ is a 1-Lipschitz map. Let $p: B_{d}^{n-l} \times B_{d}^{n} \rightarrow B_{d}^{2 n-d}$ be the natural radial projection. We define $h=p \circ \bar{h}$. Clearly, the condition 1) holds. Now letting $y \in N$ be an arbitrary point, show that $p r_{1}(y) \in N_{2 d}(M)$. First, $y$ can be presented as $j(x)+w_{1}+w_{2}$ where $x \in M, w_{1} \in B_{\delta}^{n-l} \cong \nu_{1}^{-1}(j(x)), \nu_{1}: N_{\delta}^{\prime} \rightarrow j(M)$ is the natural projection, and $w_{2} \in B_{d}^{n} \subset L^{\perp}$. Then $\operatorname{pr}_{1}\left(j(x)+w_{1}+w_{2}\right)=$ $p r_{1}(j(x))+p r_{1}\left(w_{1}\right)+p r_{1}\left(w_{2}\right)=x+u+p r_{1} w_{2}$ where $w_{1}=\bar{j}(u)=(u, \mu u)$ and $u$ is a normal vector to $M$ at point $x$ of the length $\leq \epsilon$. Then $\left\|\operatorname{pr}_{1}(y)-x\right\|=$ $\left\|u+p r_{1}\left(w_{2}\right)\right\| \leq\|u\|+\left\|w_{2}\right\| \leq \epsilon+d \leq 2 d$. Hence $\operatorname{pr}_{1}(y) \in N_{2 d}(M)$.

Proof of Theorem 1. Let $\operatorname{dim} X=k$. As we agreed, there is a homeomorphism $h: \mathbf{R}^{k} \rightarrow X$. Let $g: X \rightarrow \mathbf{R}^{n}$ be a large scale uniform embedding. Since $n>2 k$, the map $g$ admits an approximation $g^{\prime}: X \rightarrow \mathbf{R}^{n}$ by a smooth embedding with $\left\|g(x)-g^{\prime}(x)\right\|<1$ for all $x \in X$. Clearly, in the coarse category, the space $X$ is equivalent to the space $g^{\prime}(X)$ supplied with the metric from $\mathbf{R}^{n}$. From now on we will identify $X$ with $g^{\prime}(X)$.

For every $d>0$, we construct a codimension-zero submanifold $V \subset X \times \mathbf{R}^{n}$ and a 1-Lipschitz map of degree one $f:(V, \partial V) \rightarrow\left(B_{d}^{k+n}, \partial B_{d}^{k+n}\right)$. Clearly, this would imply the integral hypersphericity of $X \times \mathbf{R}^{n}$. We apply Lemma 1 to $X$ embedded in $W=\mathbf{R}^{n}$. Then by Lemma 1 , for large enough $r$, there is a retraction $\alpha$ of the $2 d$-neighborhood $N_{2 d}\left(h\left(S_{r}^{k-1}\right)\right)$ in $\mathbf{R}^{n}$ onto a curved $(k-1)$-sphere $h\left(S_{r}^{k-1}\right)$. We may require that $\alpha$ as well as $h$ be smooth maps. We assume that $\mathbf{R}^{n} \subset S^{n}$ is compactified to the $n$-sphere and assume that $f: S^{n} \rightarrow B_{r}^{k}$ is a smooth extension of $h^{-1} \circ \alpha$. Let $x_{0}$ be a regular value of $f$ and assume that $f(\infty) \neq x_{0}$. Then the fiber $M=f^{-1}\left(x_{0}\right)$ is a closed $(n-k)$-dimensional manifold that admits a trivial tubular neighborhood $N_{\epsilon}(M)$ for some $\epsilon>0$. We may assume that $\epsilon<d$. Then we apply Lemma 2 to obtain an embedding $j: M \rightarrow \mathbf{R}^{n} \times \mathbf{R}^{n}$ with a regular neighborhood $N$ having the properties (1) and (2). In view of the condition (2) of Lemma 2, for large enough $R$, the neighborhood $N$ is contained in $N_{2 d}(M) \times B_{R}^{n}$. Hence the boundary $\partial\left(h\left(B_{r}^{k}\right) \times B_{R}^{n}\right)=h\left(S_{r}^{k-1}\right) \times B_{R}^{n} \cup h\left(B_{r}^{k}\right) \times \partial B_{R}^{n}$ does not intersect $N$. Note that the manifold $M$ is linked in $\mathbf{R}^{n}$ with $h\left(S_{r}^{k-1}\right)$ with the linking number one. Also, $M$ is linked with $\partial\left(h\left(B_{r}^{k}\right) \times B_{R}^{n}\right)$ with the linking number one. 
Since $j(M)$ is homotopic to $M$ inside $N_{2 d}(M) \times \operatorname{Int}\left(B_{R}^{n}\right)$, it follows that $j(M)$ is linked with $\partial\left(h\left(B_{r}^{k}\right) \times B_{R}^{n}\right)$ with the linking number one. Consider the intersection $V=\left(X \times \mathbf{R}^{n}\right) \cap N$. We may assume that $V$ is a manifold with boundary. Since $\partial V$ is homologous to $\partial\left(h\left(B_{r}^{k}\right) \times B_{R}^{n}\right)$ in $\left(\mathbf{R}^{n} \times \mathbf{R}^{n}\right) \backslash N$, the linking number of $\partial V$ and $j(M)$ is one. Since the intersection number of $V$ and $j(M)$ is one, the restriction $\left.h\right|_{V}:(V, \partial V) \rightarrow\left(B_{d}^{n+k}, \partial B_{d}^{n+k}\right)$ of a 1-Lipschitz map $h: N \rightarrow B_{d}^{n+k}$ from Lemma 2 has degree equal to one.

Proof of Theorem 2. Let $\operatorname{dim} X=k$. Let $K$ be a triangulation on $X$ with diameters of simplexes $\leq 1$. We change the original embedding of $X$ into $l_{2}$ to a piecewise linear one that agrees with it on the vertices. We present $X$ as a union of finite subcomplexes: $X=\bigcup K_{i}, K_{i} \subset K_{i+1}$. Then every complex $K_{i}$ lies in a finitedimensional Euclidean space $\mathbf{R}_{i}^{n} \subset l_{2}$. Let $d$ be given. As in the proof of Theorem 1 , we construct a submanifold $V \subset X \times \mathbf{R}^{n(d)}$ and a short map of degree one $f:(V, \partial V) \rightarrow\left(B_{d}^{k+n(d)}, \partial B_{d}^{k+n(d)}\right)$. By Lemma 1, for large enough $r$, there is a retraction $\alpha$ of the $2 d$-neighborhood $N_{2 d}\left(h\left(S_{r}^{k-1}\right)\right)$ in $l_{2}$ onto $h\left(S_{r}^{k-1}\right)$. There is $i$ such that $h\left(S_{r}^{k-1}\right) \subset K_{i}$. Then we can work in $\mathbf{R}^{n_{i}}$ as in the proof of Theorem 1, and we get $n(d)=n_{i}$.

Remark. If we replace in the above argument the Hilbert space $l_{2}$ by the Banach space $l_{\infty}$, we will obtain the following condition on $X$ : For every $\epsilon>0$, there exist $m$ and a submanifold with boundary $W \subset X \times \mathbf{R}^{m}$ that admits an $\epsilon$-contracting map onto the $l_{\infty}$ unit ball $f:(W, \partial W) \rightarrow\left(B_{\infty}^{n+m}, \partial B_{\infty}^{n+m}\right)$ of degree one. It is unclear if it would be possible to get a stable hypersphericity of $X$ from this (see [G4], page 8).

\section{Embedding TheOREM For ASYMPTOTIC DIMENSION}

We recall that the asymptotic $\operatorname{dimension} \operatorname{asdim} X$ of a metric space $X$ is the least number $n$, if there is such a number, such that for any $d>0$, there is a uniformly bounded cover $\mathcal{U}$ of $X$ that consists of $n+1 d$-disjoint families $\mathcal{U}=\mathcal{U}^{0} \cup \cdots \cup \mathcal{U}^{n}$. A family of sets $\mathcal{V}$ is $d$-disjoint if $d\left(V, V^{\prime}\right)=\inf \left\{d\left(x, x^{\prime}\right) \mid x \in V, x^{\prime} \in V^{\prime}, V, V^{\prime} \in \mathcal{V}\right\}>$ $d$. By $N_{r}(A)$ we denote the $r$-neigborhood of $A \subset X$ in a metric space $X$ if $r>0$, and if $r \leq 0$ we define $N_{r}(A)=A \backslash N_{-r}(X \backslash A)$. Let mesh $(\mathcal{U})$ denote an upper bound for diameters of elements of a cover $\mathcal{U}$ and let $L(\mathcal{U})$ denote the Lebesgue number of $\mathcal{U}$.

Proposition 1. If a metric space $X$ with a base point $x_{0}$ has $\operatorname{asdim}(X) \leq n$, then there is a sequence of uniformly bounded open covers $\mathcal{U}_{k}$ of $X$ such that each cover $\mathcal{U}_{k}$ splits into a collection of $n+1 d_{k}$-disjoint families $\mathcal{U}_{k}=\mathcal{U}_{k}^{0} \cup \cdots \cup \mathcal{U}_{k}^{n}$ having the properties:

(1) $L\left(\mathcal{U}_{k}\right)>d_{k}$ and $N_{-d_{k}}(U) \neq \emptyset$ for all $U \in \mathcal{U}_{k}$;

(2) $d_{k}=2^{k+1} m_{k-1}$ where $m_{k-1}=\operatorname{mesh}\left(\mathcal{U}_{k-1}\right)$;

(3) for any $m \in \mathbf{N}$ and every $i \in\{0, \ldots, n\}$, there is $l$ and $U \in \mathcal{U}_{l}^{i}$ such that $N_{-d_{l}}(U) \supset B_{m}\left(x_{0}\right)$;

(4) for every $i \in\{0, \ldots, n\}$ and $k<l$, for any $U \in \mathcal{U}_{k}^{i}$ and $V \in \mathcal{U}_{l}^{i}$ with $U \not \subset V$, there is the inequality $d(U, V) \geq 4$.

Proof. We construct it by induction on $k$. We start with a cover $\mathcal{U}_{0}$ with $d_{0}>2$ and enumerate the partition $\mathcal{U}_{0}=\mathcal{U}_{0}^{0} \cup \cdots \cup \mathcal{U}_{0}^{n}$ in such a way that $d\left(x_{0}, X \backslash U\right)>d_{0}$ for some $U \in \mathcal{U}_{k}^{0}$. We formulate condition (3) in a concrete fashion: 
$(3)^{\prime}$ For $l=m(n+1)+i$ where $0 \leq i \leq n$, there is $U \in \mathcal{U}_{l}^{i}$ such that $N_{-d_{l}}(U) \supset$ $B_{m}\left(x_{0}\right)$.

Assume that the family $\left\{\mathcal{U}_{k}\right\}$ is constructed for all $k \leq l$ such that the conditions (1), (2), $(3)^{\prime},(4)$ hold. We define $d_{l+1}=2^{l+2} m_{l}$ and consider a uniformly bounded cover $\overline{\mathcal{U}}_{l+1}$ with the Lebesgue number $L\left(\overline{\mathcal{U}}_{l+1}\right)>2 d_{l+1}$ and with splitting in $n+1$ $d_{l+1}$-disjoint families $\overline{\mathcal{U}}_{l+1}=\overline{\mathcal{U}}_{l+1}^{0} \cup \cdots \cup \overline{\mathcal{U}}_{l+1}^{n}$. Then we may assume that for all elements $U \in \overline{\mathcal{U}}_{l+1}$, we have $N_{-2 d_{l+1}}(U) \neq \emptyset$. Indeed, we can delete all elements $U$ from the cover $\overline{\mathcal{U}}_{l+1}$ that do not satisfy this property. Then, since $L\left(\overline{\mathcal{U}}_{l+1}\right)>2 d_{l+1}$, we still will have a cover of $X$. We enumerate families $\overline{\mathcal{U}}_{l+1}^{0} \cup \cdots \cup \overline{\mathcal{U}}_{l+1}^{n}$ in such a way that $d\left(x_{0}, X \backslash U\right)>2 d_{l+1}$ for some $U \in \overline{\mathcal{U}}_{l+1}^{i}$ for $i=l+1 \bmod n+1$. For every $U \in \overline{\mathcal{U}}_{l+1}^{i}$ we define $\tilde{U}=U \backslash \bigcup_{V \not \subset U ; V \in \mathcal{U}_{k}^{i}, k \leq l} \overline{N_{4}(V)}$. We define $\mathcal{U}_{l+1}^{i}=\{\tilde{U} \mid$ $\left.U \in \overline{\mathcal{U}}_{l+1}^{i}\right\}$. Next we verify all the properties.

(1). Take a point $x \in X$. Then there exists $U \in \overline{\mathcal{U}}_{l+1}$ such that $B_{2 d_{l+1}}(x) \subset U$. Then $B_{d_{l+1}}(x) \subset U \backslash N_{d_{l+1}}(X \backslash U) \subset U \backslash N_{m_{l}+4}(X \backslash U) \subset \tilde{U}$. Note that $N_{-d_{l+1}}(\tilde{U})=$ $\tilde{U} \backslash N_{d_{l+1}}(X \backslash U) \supset U \backslash N_{d_{l+1}+m_{l}+4}(X \backslash U) \supset N_{2 d_{l+1}}(U)=N_{-2 d_{l+1}}(U) \neq \emptyset$.

(2). This condition holds by the definition.

$(3)^{\prime}$. This condition holds by the construction.

(4). Assume that $V \in \mathcal{U}_{k}^{i}, k \leq l$ and $V \not \subset \tilde{U}$. If $V \not \subset U$, then $d(V, \tilde{U}) \geq 4$. Assume that $V \subset U$. Since $V \not \subset \tilde{U}$, there exists $V^{\prime} \not \subset U$ and $V^{\prime} \in \mathcal{U}_{s}^{i}$ for some $s \leq l$ such that $V \cap N_{4}\left(V^{\prime}\right) \neq \emptyset$. Hence $d\left(V, V^{\prime}\right)<4$. By condition (4) and the induction assumption, we have either $V \subset V^{\prime}$ or $V^{\prime} \subset V$. In the first case, it follows that $d(V, \tilde{U}) \geq d\left(V^{\prime}, \tilde{U}\right) \geq 4$. The second case is impossible, since $V^{\prime} \subset V \subset U$ contradicts the fact that $V^{\prime} \not \subset U$.

A metric space $(X, d)$ is called a geodesic metric space if for every pair of points $x, y \in X$, there is an isometric embedding of the interval $\xi:[0, d(x, y)] \rightarrow X$ with $\xi(0)=x$ and $\xi(d(x, y))=y$. The map $\xi$ is called a geodesic in $X$ joining $x$ and $y$.

Proposition 2. Let $W$ be an open set in a geodesic metric space $X$ and let $f$ : $X \rightarrow Y$ be a map between metric spaces such that the restrictions $\left.f\right|_{W}$ and $\left.f\right|_{X \backslash W}$ are $\lambda$-Lipschitz. Then $f$ is $\lambda$-Lipschitz.

Proof. Note that the restriction to the closure $\left.f\right|_{\bar{W}}$ is also $\lambda$-Lipschitz. Let $x, y \in$ $X, x \in W, y \in X \backslash W$, be two points and let $\xi: I=[0, d(x, y)] \rightarrow X$ be a geodesic between them. Since the interval $I$ is connected, we have $\xi^{-1}(\partial W) \neq \emptyset$. Let $\xi(t)=z \in \partial W$. Then $d_{Y}(f(x), f(y)) \leq d_{Y}(f(x), f(z))+d_{Y}(f(z), f(y)) \leq$ $\lambda d(x, z)+\lambda d(z, y)=\lambda t+\lambda(d(x, y)-t)=\lambda d(x, y)$.

Theorem 3. Assume that $X$ is a geodesic metric space of bounded geometry with $\operatorname{asdim}(X) \leq n$. Then $X$ can be large scale uniformly embedded in the product of $n+1$ locally finite trees.

Proof. Let $\mathcal{U}_{k}$ be a sequence of covers of $X$ from Proposition 1. Let $\mathcal{V}_{i}=\coprod_{k} \mathcal{U}_{k}^{i}$. We define a map $\psi: \mathcal{V}_{i} \rightarrow \mathcal{V}_{i}$ by the following rule: $\psi(V)$ is the smallest $U \in$ $\mathcal{V}_{i}$ with respect to the inclusion such that $V \neq U$ and $V \subset U$. The conditions 3) and 4) of Proposition 1 and disjointness of $\mathcal{U}_{k}^{i}$ for all $k$ imply that $\psi$ is welldefined. For every $i \in\{0, \ldots, n\}$, construct an oriented graph $T^{i}$ as follows. For every $U \in \mathcal{U}_{k}^{i}$, we consider an interval $I_{U}$ isometric to $\left[0,2^{k}\right]$ and oriented from $2^{k}$ to 0 . For every $V \in \psi^{-1}(U)$ we attach $I_{V}$ by the 0 -end to an integer point 
$a_{V}=\min \left\{2^{k},\left[\sup \phi_{U}(V) \frac{1}{2 m_{k-1}}\right]\right\}$ of $I_{U}$ where $\phi_{U}(x)=d(x, X \backslash U), \sup \phi_{U}(V)=$ $\sup _{v \in V} \phi_{U}(v)$, and $[a]$ means the integer part of $a$.

We show that the graph $T^{i}$ is a locally finite tree. For every $U, V \in \mathcal{V}_{i}$, by the property 3), there exists $W \in \mathcal{V}_{i}$ such that $U \cup V \subset W$. This implies the connectedness of $T^{i}$. Since the orientation on $T^{i}$ defines a flow, i.e., every vertex is an initial point only for one arrow, it follows that, every cycle in $T^{i}$ must be oriented. By the construction, every vertex $v$ in $T^{i}$ corresponds to an integer nonzero point from the interval $I_{U}$ for some $U \in \mathcal{U}_{k}^{i}$ for some $k$. We define that number $k$ to be called the level of this vertex: $k=l(v)$. For every edge in $T^{i}$, the level of the initial point does not exceed the level of the terminal point: $l(o) \leq l(t)$. Therefore, all vertices in an oriented cycle must have the same level. This implies that all vertices of that cycle lie in one interval $I_{U}$. Therefore there are no cycles in $T^{i}$. Thus, $T^{i}$ is a tree. Note that for every nonzero vertex in $I_{U}$, only finitely many intervals are attached to it. This implies that if a vertex $v$ in $T^{i}$ is of infinite order. Then $v$ must be the 0 -vertex for all intervals involved. So a vertex $v$ of infinite order defines an infinite sequence $U_{1} \subset U_{2} \subset \cdots \subset U_{m} \subset$ with $\psi\left(U_{j}\right)=U_{j+1}$ and $a_{U_{j}}=0$ for all $j$. Let $U_{j} \in \mathcal{U}_{k_{j}}^{i}$. Then $\frac{1}{2 m_{k_{j}-1}} d\left(x, X \backslash U_{j}\right)<1$ for all $x \in U_{j-1}$. Hence $d\left(x, X \backslash U_{j}\right)<d_{k_{j}}$ for all $x \in U_{j-1}$, i.e., $U_{j-1} \cap N_{-d_{k_{j}}}\left(U_{j}\right)=\emptyset$ for all $j$. By the property 3) from Proposition 1, there is $U \in \mathcal{U}_{l}^{i}$ with $N_{-d_{l}}(U) \supset U_{1}$. Then the condition 4) implies that $U=U_{j}$ for some $j$, whence $l=k_{j}$. Note that the condition $N_{-d_{l}}\left(U_{j}\right) \supset U_{1}$ implies that $U_{j-1} \cap N_{-d_{l}}\left(U_{j}\right) \neq \emptyset$. This contradiction implies that there are no vertices of infinite order in the tree $T^{i}$.

Next we define a map $p_{i}: X \rightarrow T^{i}$ as the union of maps

$$
p_{i}^{k}: \bigcup_{U \in \mathcal{U}_{k}^{i}} U \rightarrow T^{i}
$$

by induction on $k$.

First we define $\left.p_{i}^{0}\right|_{V}: V \rightarrow I_{V}=[0,1]$ by the formula $p_{i}^{0}(x)=\min \{1, d(x, X \backslash V)\}$.

We assume that $p_{i}^{k-1}$ is defined and it is 1-Lipschitz. For every $U \in \mathcal{U}_{k}^{i}$, we define a map

$$
\xi: \bar{N}_{-d_{k}}(U) \cup \partial U \cup \bigcup_{V \in \psi^{-1}(U)} \partial V \rightarrow I_{U}=\left[0,2^{k}\right]
$$

by the rule: $\xi\left(\bar{N}_{-d_{k}}(U)\right)=2^{k}, \xi(\partial U)=0$ and $\xi(\partial V)=a_{v}$. We shall show that $\xi$ is a 1-Lipschitz map. Let $y \in \partial V$ and $z \in \partial V^{\prime}, V, V^{\prime} \in \psi^{-1}(U)$ and $V \neq V^{\prime}$. Then by the condition 4$), d(y, z) \geq 4$.

We recall that $\phi_{U}(x)=d(x, X \backslash U)$ and $\sup \phi_{U}(V)=\sup _{v \in V} \phi_{U}(v)$. Let $\sup \phi_{U}(V)=\phi_{U}\left(y^{*}\right)$ and $\sup \phi_{U}\left(V^{\prime}\right)=\phi_{U}\left(z^{*}\right)$, where $y^{*} \in \bar{V}$ and $z^{*} \in \bar{V}^{\prime}$.

Then

$$
\begin{aligned}
& |\xi(y)-\xi(z)|=\left|a_{V}-a_{V^{\prime}}\right| \leq \frac{1}{2 m_{k-1}}\left|\phi_{U}\left(y^{*}\right)-\phi_{U}\left(z^{*}\right)\right|+1 \\
& \quad \leq \frac{1}{2 m_{k-1}}\left(\left|\phi_{U}\left(y^{*}\right)-\phi_{U}(y)\right|+\left|\phi_{U}(y)-\phi_{U}(z)\right|+\left|\phi_{U}\left(z^{*}\right)-\phi_{U}(z)\right|\right)+1 \\
& \quad \leq \frac{1}{2 m_{k-1}}\left(\left|\phi_{U}\left(y^{*}\right)-\phi_{U}(y)\right|+\left|\phi_{U}(y)\right|+\left|\phi_{U}(z)\right|+\left|\phi_{U}\left(z^{*}\right)-\phi_{U}(z)\right|\right)+1 \\
& \quad \leq \frac{1}{2 m_{k-1}}\left|m_{k-1}+d(y, z)+m_{k-1}\right|+1=\frac{1}{2 m_{k-1}} d(y, z)+2 \leq d(y, z) .
\end{aligned}
$$


Here we applied condition 4).

Now let $y \in \partial V$ and $z \in \partial U$. Then $|\xi(y)-\xi(z)|=a_{V}$. If $a_{V} \neq 0$, then $\frac{1}{2 m_{k-1}} \phi_{U}\left(y^{*}\right) \geq 1$. Hence $\frac{1}{2 m_{k-1}}\left(\phi_{U}(y)+m_{k-1}\right) \geq 1$. This implies that $\phi_{U}(y) \geq$ $m_{k-1}$ and, hence, $d(y, z) \geq m_{k-1}$. Then

$$
a_{V} \leq \frac{1}{2 m_{k-1}}\left(d(y, X \backslash U)+m_{k-1}\right) \leq \frac{1}{2 m_{k-1}} d(y, z)+\frac{1}{2} \leq d(y, z) .
$$

The case when $y \in \partial U$ and $z \in \bar{N}_{-d_{k}}(U)$ is obvious.

If $y \in \partial V$ and $z \in \bar{N}_{-d_{k}}(U)$, then

$$
\begin{aligned}
|\xi(z)-\xi(y)| & \leq 2^{k}-\frac{1}{2 m_{k-1}}\left(d(y, X \backslash U)-m_{k-1}\right)+1 \\
& \leq \frac{2^{k}}{d_{k}}\left(d_{k}-d(y, X \backslash U)\right)+2 \\
& \leq \frac{2^{k}}{d_{k}}(d(z, X \backslash U)-d(y, X \backslash U))+2 \leq d(y, z) .
\end{aligned}
$$

There exists a 1-Lipschitz extension $\xi_{U}^{\prime}: U \backslash \bigcup_{V \in \psi^{-1}(U)} V \rightarrow I_{U}$ of the map $\xi$. By the induction assumption for all $V \in \psi^{-1}(U)$, there are 1-Lipschitz maps $\bar{\xi}_{V}=\left.p_{i}^{k-1}\right|_{V}: V \rightarrow T_{V}$, where $T_{V} \subset T^{i}$ consists of the points of $T^{i}$ that can be taken to $I_{V}$ by the orientation flow. By Proposition 2, the union

$$
\bar{\xi}_{U}=\xi_{U}^{\prime} \cup \bigcup_{V \in \psi^{-1}(U)} \bar{\xi}_{V}: U \rightarrow I_{U} \cup \bigcup_{V \in \psi^{-1}(U)} T_{V}=T_{U}
$$

is 1-Lipschitz. We define $\left.p_{i}^{k}\right|_{U}=\bar{\xi}_{U}$.

We shall show that the diagonal product $p=\Delta p_{i}: X \rightarrow \prod T^{i}$ is a large scale uniform embedding. We consider the $l_{1}$-metric on $\prod T^{i}$. Since each map $p_{i}$ is 1-Lipschitz, the map $p$ is Lipschitz. Clearly, $\operatorname{Dist}\left(p(x), p\left(x^{\prime}\right)\right) \geq \rho\left(d\left(x, x^{\prime}\right)\right)$ for the function $\rho(t)=\inf \left\{\operatorname{Dist}\left(p(x), p\left(x^{\prime}\right)\right) \mid d\left(x, x^{\prime}\right) \geq t\right\}$. Assume that $\rho$ is bounded from above. Then there is a sequence of pairs $\left(x_{k}, x_{k}^{\prime}\right)$ of points with $d\left(x_{k}, x_{k}^{\prime}\right)>m_{k}$ and with $\operatorname{Dist}\left(p\left(x_{k}\right), p\left(x_{k}^{\prime}\right)\right) \leq b$ for all $k$. For any $k$ there is an element $U \in \mathcal{U}_{k}^{i}$ such that $d\left(x_{k}, X \backslash U\right)>d_{k}$. Since $d\left(x_{k}, x_{k}^{\prime}\right)>m_{k}$, it follows that $x_{k}^{\prime} \notin U$. Note that $\bar{\xi}_{U}\left(x_{k}\right)=2^{k}$ and hence the distance between $p_{i}\left(x_{k}\right)$ and $p_{i}\left(x_{k}^{\prime}\right)$ in the graph $T^{i}$ is greater than $2^{k}$. Therefore, $\operatorname{Dist}\left(p\left(x_{k}\right), p\left(x_{k}^{\prime}\right)\right) \geq 2^{k}$. Therefore $\rho$ tends to infinity and $p$ is a uniform embedding.

Remark. It was investigated in [DZ] when the trees in Theorem 3 can be required to be binary trees.

Lemma 3. Every locally finite tree is large scale uniformly embeddable in a complete simply connected 2-dimensional manifold $K(M)$ with negative curvature: $K(M) \leq-k_{2}<0$.

Proof. Let $T$ be a tree. Fix a vertex $t_{0} \in T$. Note that $T$ is supplied with a geodesic metric such that every edge has length one. Without loss of generality, we may assume that every vertex in $T$ has valency $\geq 2$. By induction on $n$, one can construct a topological embedding of $T$ in the Euclidean plane $\mathbf{R}^{2}$ in such a way that $t_{0}$ is identified with 0 , for every $n \in \mathbf{N}$ the metric sphere in $T$ of radius $n$ centered at $t_{0}, S(n)=\left\{x \in T \mid d\left(T, t_{0}\right)=n\right\}$, lies in the circle $S(n)=\left\{x \in \mathbf{R}^{2} \mid\|x\|=n\right\}$ and every edge of $T$ is mapped linearly to $\mathbf{R}^{2}$. 
We shall show that the boundary of every component $C$ of the complement $\mathbf{R}^{2} \backslash T$ is homeomorphic to the real line $\mathbf{R}$. The condition on valency implies that every edge in $T$ can be extended to a ray in either direction. This implies that for every vertex $t$ with the valency $v(t)$, there are exactly $v(t)$ components in $\mathbf{R}^{2} \backslash T$ having $t$ as a boundary point. Therefore, the boundary $\partial C$ cannot contain three edges meeting at one point. Since $T$ is connected, the Alexander duality implies that every component $C$ is simply connected and hence its boundary $\partial C$ is connected. Since $\mathbf{R}^{2} \backslash T$ has more than one component, $\partial C$ separates the plane. All this implies that $\partial C$ is homeomorphic to $\mathbf{R}$. The boundary $\partial C \subset T$ with the induced metric from the tree is naturally isometric to $\mathbf{R}$. For every component $C$, we attach a hyperbolic half-plane $H_{+}$along an isometry of the boundaries $\partial H_{+}$and $\partial C$. As the result, we obtain a piecewise hyperbolic manifold $M$, homeomorphic to $\mathbf{R}^{2}$, that contains the tree $T$ isometrically. The only possibility for singularities of this metric on $M$ is at vertices of the tree. We can approximate this metric by a smooth metric of strictly negative curvature.

Theorem 4. Every bounded geometry geodesic metric space $X$ with $\operatorname{asdim}(X) \leq n$ is large scale uniformly embeddable in a complete $(2 n+2)$-dimensional non-positively curved manifold $W$.

Proof. We apply Theorem 3 and Lemma 3 to obtain an embedding of $X$ into $W=\prod M_{i}$ where each $M_{i}$ is a 2-dimensional negatively curved manifold. Then $W$ is non-positively curved.

\section{On the First Theorem of Yu}

The following lemma is a generalization of Lemma 2.

Lemma 4. Let $M$ be a closed l-dimensional manifold smoothly embedded in a nonpositively curved $n$-manifold $W$ with a trivial tubular $\epsilon$-neighborhood $N_{\epsilon}(M)$. Then for any $d>0$, there is an embedding $\gamma: N_{\epsilon}(M) \rightarrow \mathbf{R}^{n}$ such that the diagonal embedding $j=(1 \Delta \gamma): M \rightarrow W \times \mathbf{R}^{n}$ has a regular neighborhood $N$ with the projection $\nu: N \rightarrow j(M)$ satisfying the conditions:

(1) there is a 1-Lipschitz map $h: N \rightarrow B_{d}^{2 n-l}$ such that the restriction $\left.h\right|_{\nu^{-1}(x)}$ : $\nu^{-1}(x) \rightarrow B_{d}^{2 n-l}$ is a homeomorphism for every $x \in j(M)$;

(2) $\operatorname{pr}_{1}(N) \subset N_{2 d}(M)$ where $p r_{1}: W \times \mathbf{R}^{n} \rightarrow W$ is the projection onto the first factor.

This Lemma together with the above Embedding Theorem allows us to prove the following:

Theorem 5. If a uniformly contractible manifold $X$ has a finite asymptotic dimension, then there is an $n$ such that $X \times \mathbf{R}^{n}$ is integrally hyperspherical.

The proof is exactly the same as in Theorem 1.

Proof of Lemma 4. Let $T W$ be the tangent bundle of a non-positively curved complete simply connected $n$-dimensional Riemannian manifold $W$ and let $\pi: T W \rightarrow$ $W$ be the projection, $\pi^{-1}(x)=T_{x}$. For every $x \in W$, there is the exponential map $e_{x}: \mathbf{R}^{n} \rightarrow W$ which takes a vector $v$ to a point $y=e_{x}(v)$ on the geodesic ray in the direction of $v$ with $d_{W}(x, y)=\|v\|$. Note that $e_{x}$ is a diffeomorphism for every $x$. The union $e=\bigcup e_{x}: T W \rightarrow W$ is also a differential map, and the diagonal product 
$\pi \Delta e: T W \rightarrow W \times W$ is a diffeomorphism. Using this, we define a trivialization $\tau: T W \rightarrow \mathbf{R}^{n}$, which takes the 0 -section $W$ to 0 , as the composition

$$
T W \stackrel{(\pi \Delta e)}{\longrightarrow} W \times W \stackrel{\alpha}{\longrightarrow} \mathbf{R}^{n} \times \mathbf{R}^{n} \stackrel{\beta}{\rightarrow} \mathbf{R}^{n},
$$

where $\alpha=e_{x_{0}}^{-1} \times e_{x_{0}}^{-1}, x_{0} \in W$ is a fixed point, and $\beta(a, b)=a-b$. Note that $\tau(x, v)=e_{x_{0}}^{-1}(x)-e_{x}^{-1} e_{x}(v)$ for $x \in M$ and $v \in T_{x}$. A tubular $\epsilon$-neighborhood of a smooth $l$-dimensional submanifold $M \subset W$ is a neighborhood $N_{\epsilon}(M)$ in $W$ such that the restriction of $e$ to $\bar{N}_{\epsilon}(M)=\bigcup_{x \in M} B_{\epsilon}^{n-l}(x) \subset T W$ is a diffeomorphism onto $N_{\epsilon}(M)$, where each $\epsilon$-ball $B_{\epsilon}^{n-l}(x) \subset T_{x}$ is taken in the normal direction for every $x \in M$.

Composing the embedding $\left.e_{x_{0}}^{-1}\right|_{N_{\epsilon}(M)}: N_{\epsilon}(M) \rightarrow \mathbf{R}^{n}$ with the multiplication by a scalar we define a $\mu$-expanding map $\gamma: N_{\epsilon}(M) \rightarrow \mathbf{R}^{n}$, where $\mu$ is a large number defined as follows. Let $q: \bar{N}_{\epsilon}(M) \rightarrow B_{\epsilon}^{n-l}$ be a smooth trivialization. Let $\lambda$ be a Lipschitz constant for the maps $q\left(\left.e\right|_{N_{\epsilon}(M)}\right)^{-1}$ and $\pi\left(\left.e\right|_{N_{\epsilon}(M)}\right)^{-1}$. We take $\mu=\frac{\lambda d}{\epsilon}$. Then we define

$$
N=\bigcup_{x \in M} \bar{B}_{2 d}(x) \times \gamma e\left(B_{\epsilon}^{n-l}(x)\right),
$$

where $\bar{B}_{r}(x)$ denotes the ball of radius $r$ in $W$ centered at $x$. Note that the condition (2) of the lemma is satisfied. Since the sets $e B_{\epsilon}^{n-l}(x)$ are disjoint for different $x$, the above union is disjoint. We can define $\nu: N \rightarrow(1 \Delta \gamma)(M)$ by the formula: $\nu\left(\left(u, \gamma e_{x}(v)\right)=(x, \gamma(x))=j(x)\right.$.

Next we define 1-Lipschitz maps $h_{1}: N \rightarrow B_{d}^{n}$ and $h_{2}: N \rightarrow B_{d}^{n-l}$ such that the sum $\left(h_{1}+h_{2}\right): N \rightarrow B_{d}^{n} \times B_{d}^{n-l}$ satisfies the condition (1). We define $h_{1}\left(\left(u, \gamma e_{x}(v)\right)=\frac{1}{2} \tau e_{x}^{-1}(u)\right.$ for $u \in \bar{B}_{2 d}(x), v \in B_{\epsilon}^{n-l}(x)$ and $x \in M$.

We define $h_{2}=\frac{d}{\epsilon} q \circ e^{-1} \circ \gamma^{-1} \circ p r_{2}$. Then we can estimate a Lipschitz constant for $h_{2}$ as the product $\frac{d}{\epsilon} \lambda \mu^{-1}=1$.

We shall show that $h_{1}$ is 1-Lipschitz. Let $z=\left(u, \gamma e_{x}(v)\right) \in W \times \mathbf{R}^{n}$ and $z^{\prime}=\left(u^{\prime}, \gamma e_{x^{\prime}}\left(v^{\prime}\right)\right) \in W \times \mathbf{R}^{n}$ be two points in $N$. Then

$$
\begin{aligned}
\left\|h_{1}(z)-h_{1}\left(z^{\prime}\right)\right\| & =\frac{1}{2}\left\|\tau e_{x}^{-1}(u)-\tau e_{x^{\prime}}^{-1}\left(u^{\prime}\right)\right\| \\
& =\frac{1}{2}\left\|e_{x_{0}}^{-1}(x)-e_{x_{0}}^{-1}(u)-e_{x_{0}}^{-1}\left(x^{\prime}\right)+e_{x_{0}}^{-1}\left(u^{\prime}\right)\right\| \\
& \leq \frac{1}{2}\left\|e_{x_{0}}^{-1}(x)-e_{x_{0}}^{-1}\left(x^{\prime}\right)\right\|+\frac{1}{2}\left\|e_{x_{0}}^{-1}(u)-e_{x_{0}}^{-1}\left(u^{\prime}\right)\right\| \\
& \leq \frac{1}{2} d\left(x, x^{\prime}\right)+\frac{1}{2} d\left(u, u^{\prime}\right) .
\end{aligned}
$$

Here we use the fact that the map $e_{x_{0}}^{-1}$ is 1-Lipschitz. Since the map $\pi\left(\left.e\right|_{N_{\epsilon}(M)}\right)^{-1}$ is $\lambda$-Lipschitz, we have $d\left(x, x^{\prime}\right) \leq \lambda d\left(e_{x}(v), e_{x^{\prime}}\left(v^{\prime}\right)\right)$ for all $v \in B_{\epsilon}^{n-l}(x)$ and $v^{\prime} \in$ $B_{\epsilon}^{n-l}\left(x^{\prime}\right)$. Since the map $\gamma^{-1}$ is $\mu^{-1}$-Lipschitz, we have $d\left(e_{x}(v), e_{x^{\prime}}\left(v^{\prime}\right)\right) \leq$ $\mu^{-1}\left\|\gamma e_{x}(v)-\gamma e_{x^{\prime}}\left(v^{\prime}\right)\right\|$. We may assume that $\frac{\epsilon}{d} \leq 1$. Then

$$
d\left(x, x^{\prime}\right) \leq \lambda \mu^{-1}\left\|\gamma e_{x}(v)-\gamma e_{x^{\prime}}\left(v^{\prime}\right)\right\| \leq\left\|\gamma e_{x}(v)-\gamma e_{x^{\prime}}\left(v^{\prime}\right)\right\| .
$$

Therefore, $\frac{1}{2}\left(d\left(x, x^{\prime}\right)+d\left(u, u^{\prime}\right)\right) \leq \sqrt{d\left(u, u^{\prime}\right)^{2}+\left\|\gamma e_{x}(v)-\gamma e_{x^{\prime}}\left(v^{\prime}\right)\right\|^{2}}=d\left(z, z^{\prime}\right)$. Then we define $h$ as the composition of $h_{1}+h_{2}$ and the natural projection of $B_{d}^{n} \times B_{d}^{n-l}$ onto $B_{d}^{2 n-l}$. The condition (1) holds true.

I am thankful to the referee of this paper for his valuable remarks. 


\section{REFERENCES}

[Dr] A. N. Dranishnikov, Asymptotic topology, Uspekhi Mat. Nauk 55 (2000) 71-116, Russian Math. Surveys 55:6 (2000), 1085-1129.

[D-F-W] A. N. Dranishnikov, S. Ferry and S. Weinberger, Large Riemannian manifolds which are flexible, Preprint (1994).

[DZ] A.N. Dranishnikov and M. Zarichnyi, Universal spaces for asymptotic dimension, Preprint (2002).

[G1] M. Gromov, Asymptotic invariants of infinite groups, Geometric Group Theory, vol. 2, London Math. Soc. Lecture Notes 182, Cambridge University Press, 1993. MR 95m:20041

[G2] M. Gromov, Large Riemannian manifolds, Lecture Notes in Math. 1201 (1986), 108-122. MR 87k:53091

[G3] M. Gromov, Positive curvature, macroscopic dimension, spectral gaps and higher signatures, Functional Analysis on the eve of the 21st century, Vol. 2, Progr. Math. 132 (1996), 1-213. MR 98d:53052

[G4] M. Gromov, Spaces and questions, Geom. Funct. Anal., Special Volume, Part 1, 2000, pp. 118-161. MR 2002e:53056

[G-L] M. Gromov and H. B. Lawson, Positive scalar curvature and the Dirac operator on complete Riemannian manifolds, Inst. Hautes Etudes Sci. Publ. Math. 58 (1983), 83196. MR 85g:58082

[H-R1] N. Higson and J. Roe, On the coarse Baum-Connes conjecture, London Math. Soc. Lecture Notes 227 (1995), 227-254. MR 97f:58127

[H-R2] N. Higson and J. Roe, Amenable group actions and the Novikov conjecture, J. Reine Angew. Math. 519 (2000), 143-153. MR 2001h:57043

[Ro1] J. Roe, Coarse cohomology and index theory for complete Riemannian manifolds, Memoirs Amer. Math. Soc. No. 497, Providence, RI, 1993. MR 94a:58193

[Ro2] J. Roe, Index theory, coarse geometry, and topology of manifolds, CBMS Regional Conference Series in Mathematics, Number 90 (1996). MR 97h:58155

[Yu1] G. Yu, The Novikov conjecture for groups with finite asymptotic dimension, Ann. of Math 147 (1998), no. 2, 325-355. MR 99k:57072

[Yu2] G. Yu, The coarse Baum-Connes conjecture for spaces which admit a uniform embedding into Hilbert space, Inventiones Mathematicae 139:1 (2000), 201-240. MR 2000j:19005

Department of Mathematics, University of Florida, 358 Little Hall, P.O. Box 118105 , Gainesville Florida 32611-8105

E-mail address: dranish@math.ufl.edu 